Supporting Information:

\title{
Melt-Processable Shape-Memory Elastomers Containing Bisurea Segments
}

\author{
Daniel M. Krajovic, ${ }^{a}$ Mitchell Anthamatten ${ }^{\mathrm{a}, *}$ \\ ${ }^{a}$ Department of Chemical Engineering, University of Rochester, 4510 Wegmans Hall P.O. 270166 \\ Rochester, NY 14627-0166, United States
}

\section{Corresponding Author}

*E-mail: mitchell.anthamatten@rochester.edu 


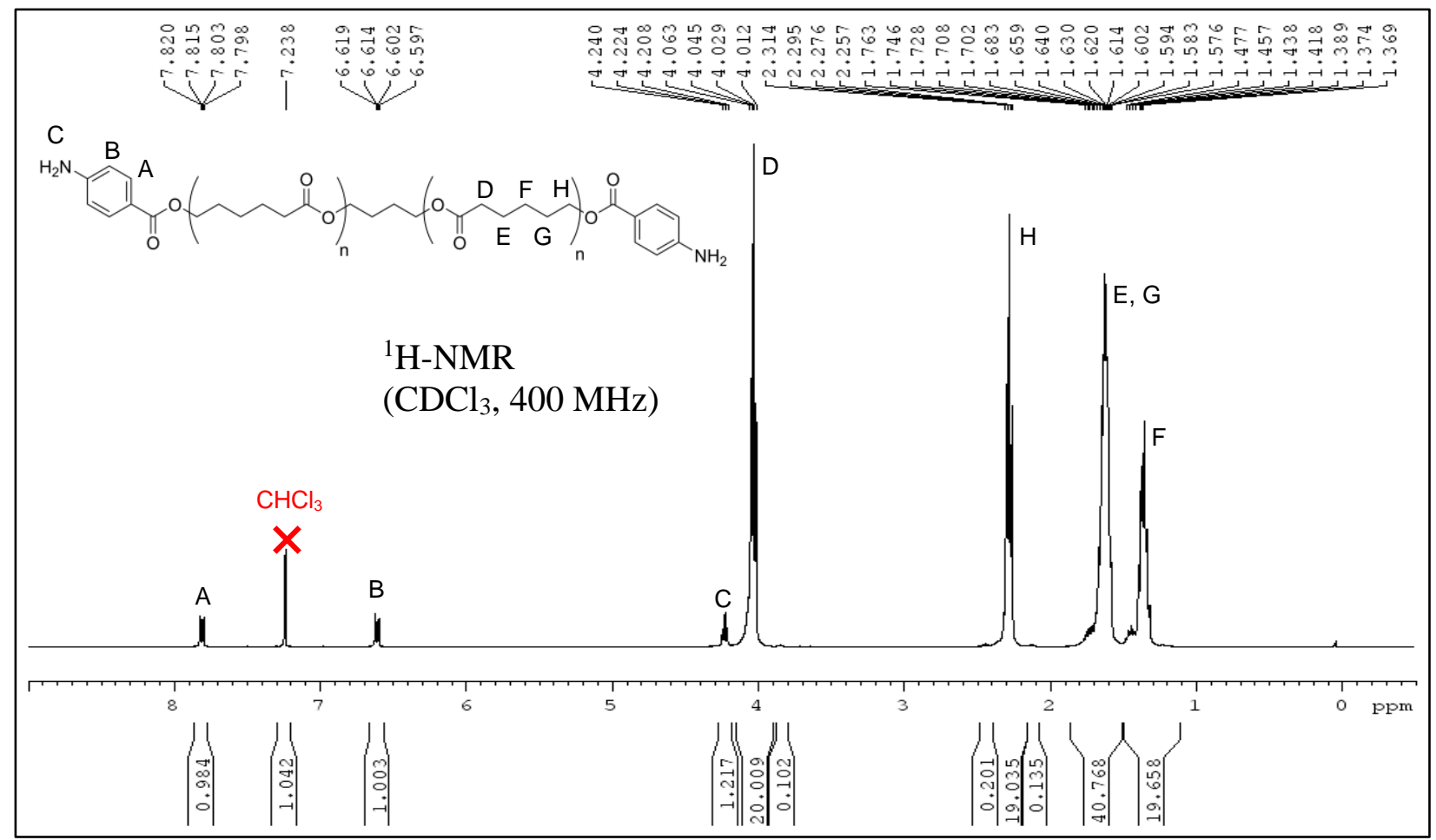

Figure S1. ${ }^{1} \mathrm{H}-\mathrm{NMR}$ spectrum of phenylamine-terminated PCL telechelic prepolymer.

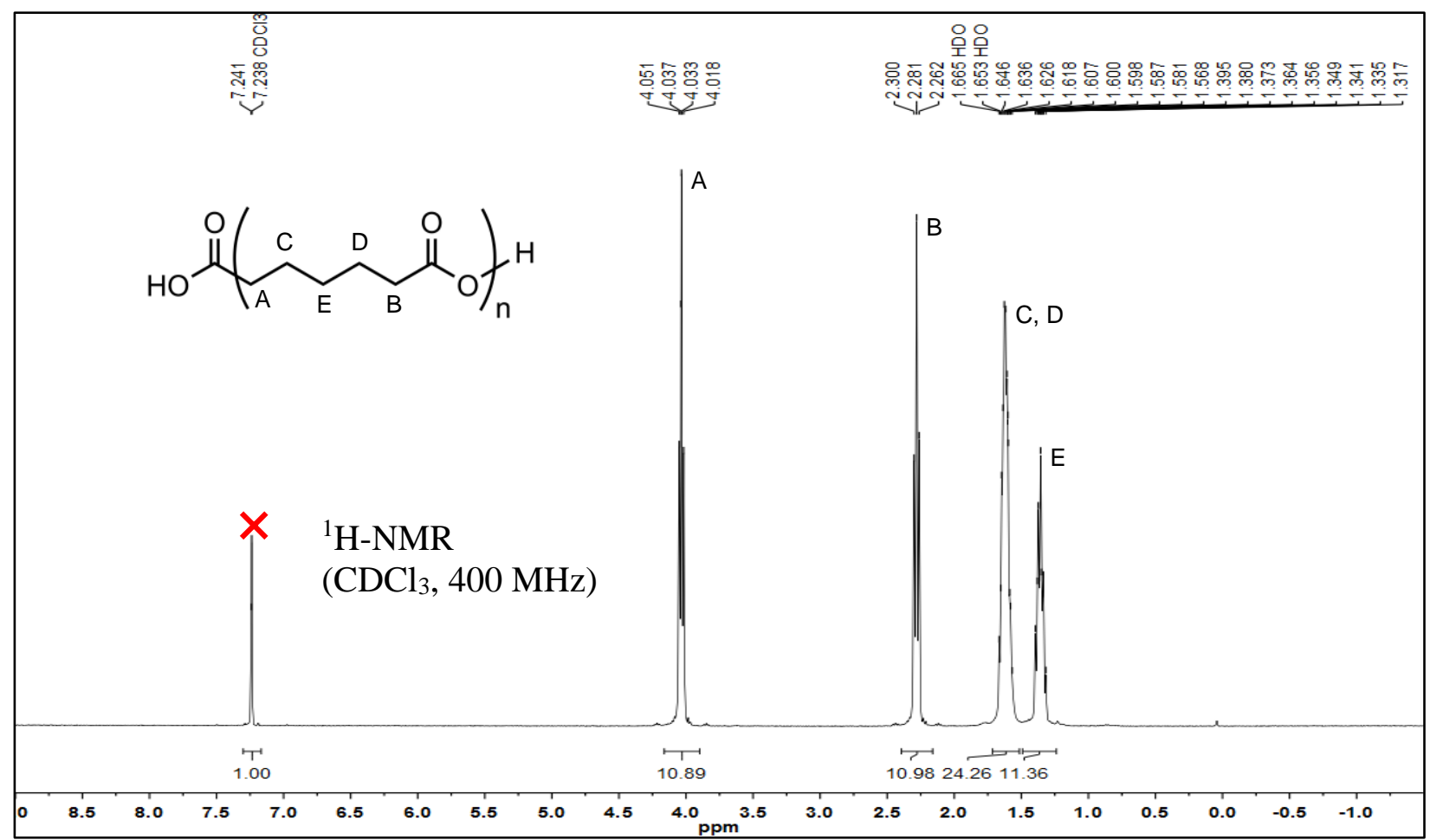

Figure S2. ${ }^{1} \mathrm{H}-\mathrm{NMR}$ spectrum of linear unfunctionalized PCL homopolymer. 


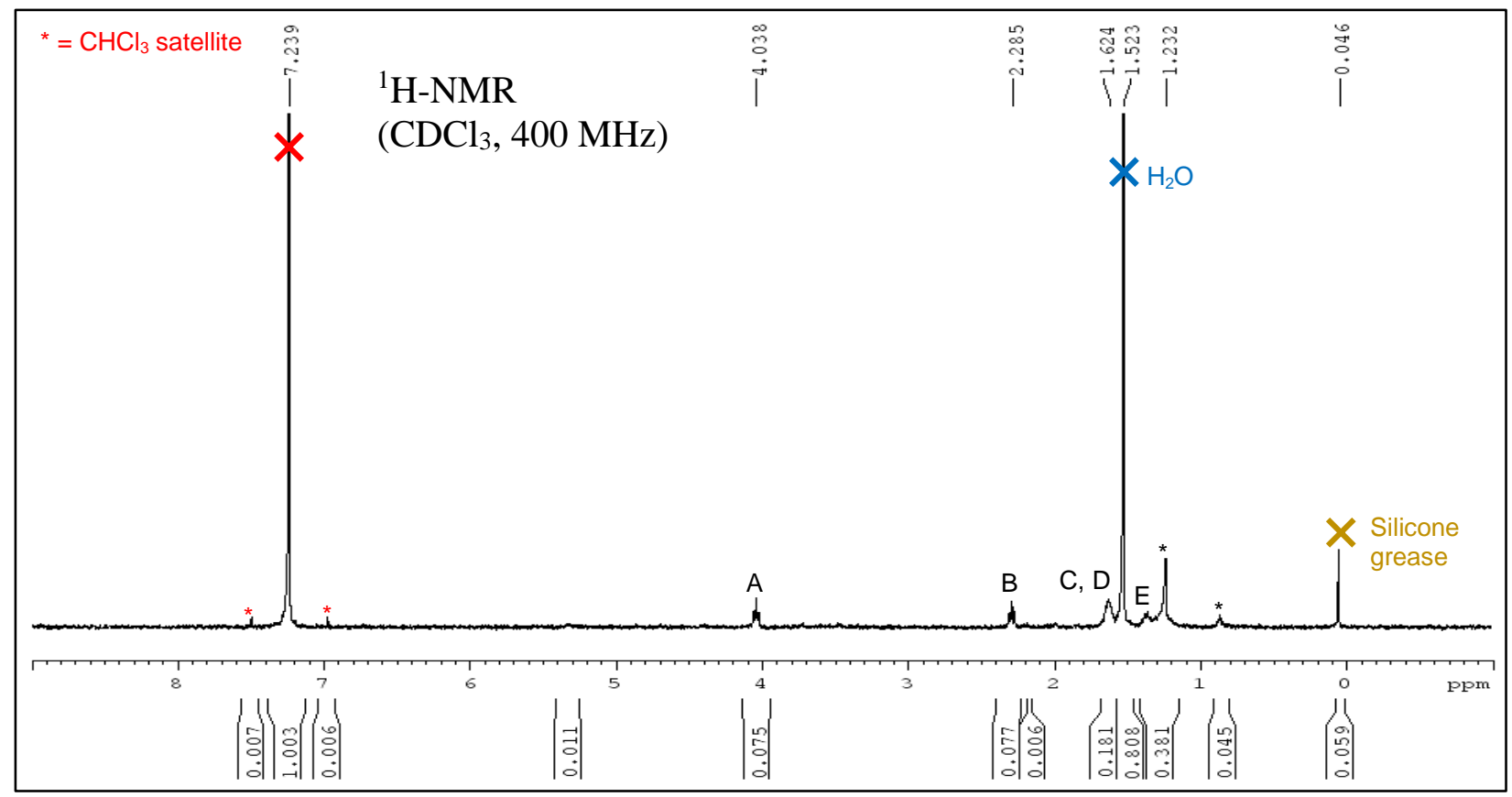

Figure S3. ${ }^{1} \mathrm{H}-\mathrm{NMR}$ spectrum of pristine PCL-TDI-BU film. See below for proton denotations. Hard segment and terminal protons' resonances are not definitively assigned. Poly(bisurea) films had best, though poor, solubility in $\mathrm{CDCl}_{3}$. The peaks with black asterisks (*) correspond to unidentified impurities.

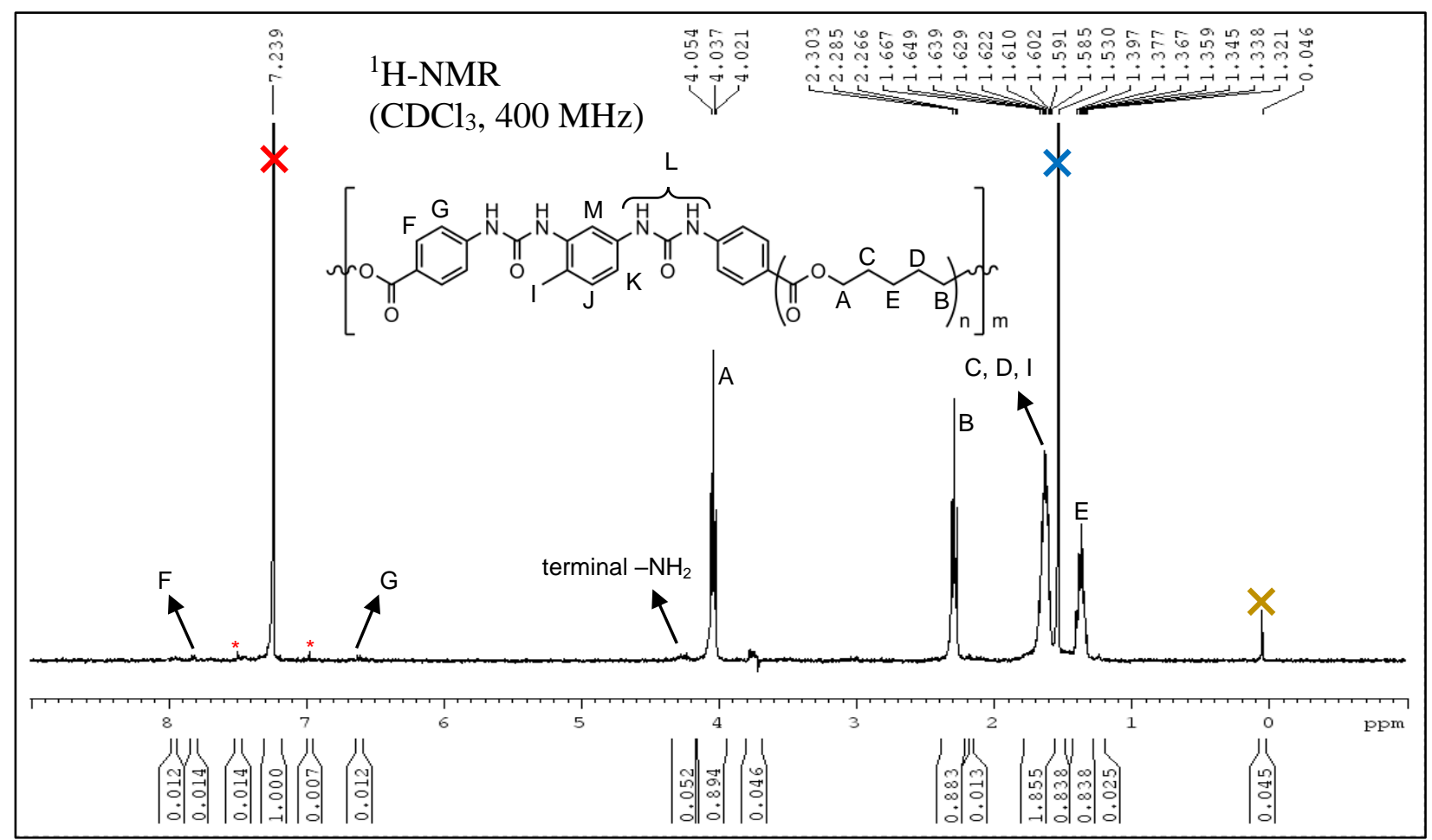

Figure S4. ${ }^{1}$ H-NMR spectrum of reprocessed PCL-TDI-BU film. Hard segment and terminal protons' resonances are not definitively assigned. 


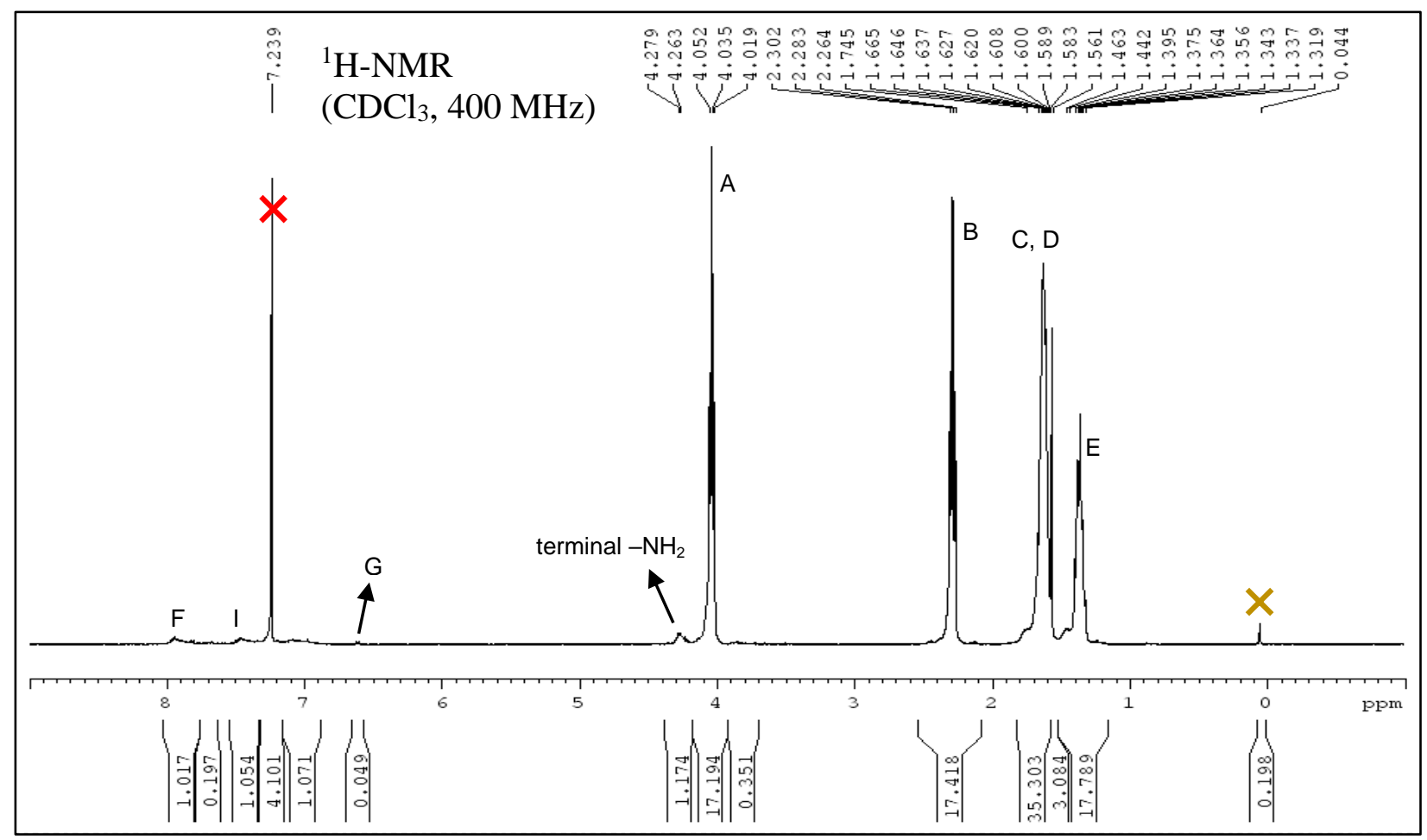

Figure S5. ${ }^{1} \mathrm{H}-\mathrm{NMR}$ spectrum of pristine PCL-MDI-BU film. See below (Figure S6) for proton denotations. Hard segment and terminal protons' resonances are not definitively assigned.

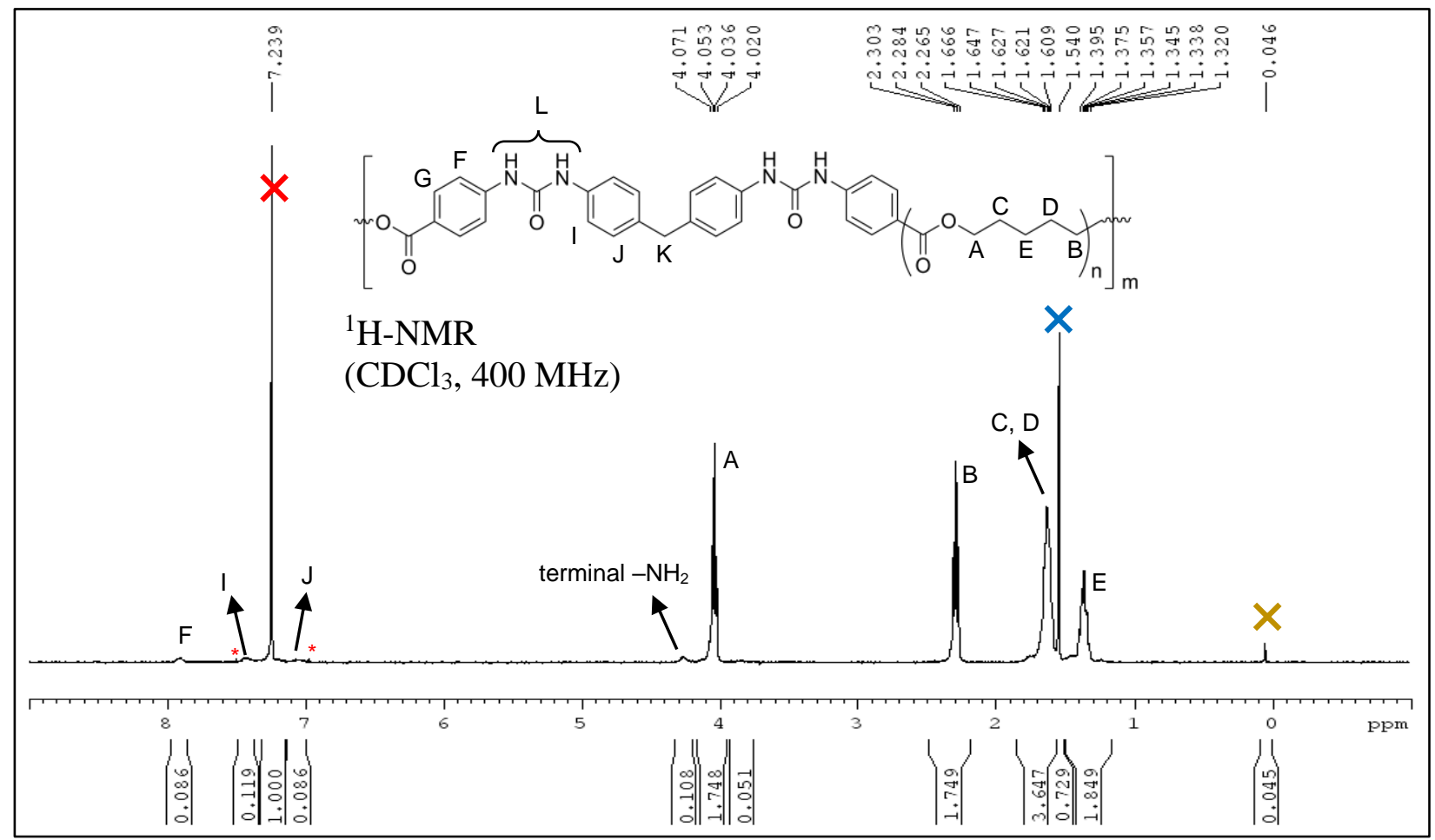

Figure S6. ${ }^{1} \mathrm{H}-\mathrm{NMR}$ spectrum of reprocessed PCL-MDI-BU film. Hard segment resonances are not definitively assigned. 


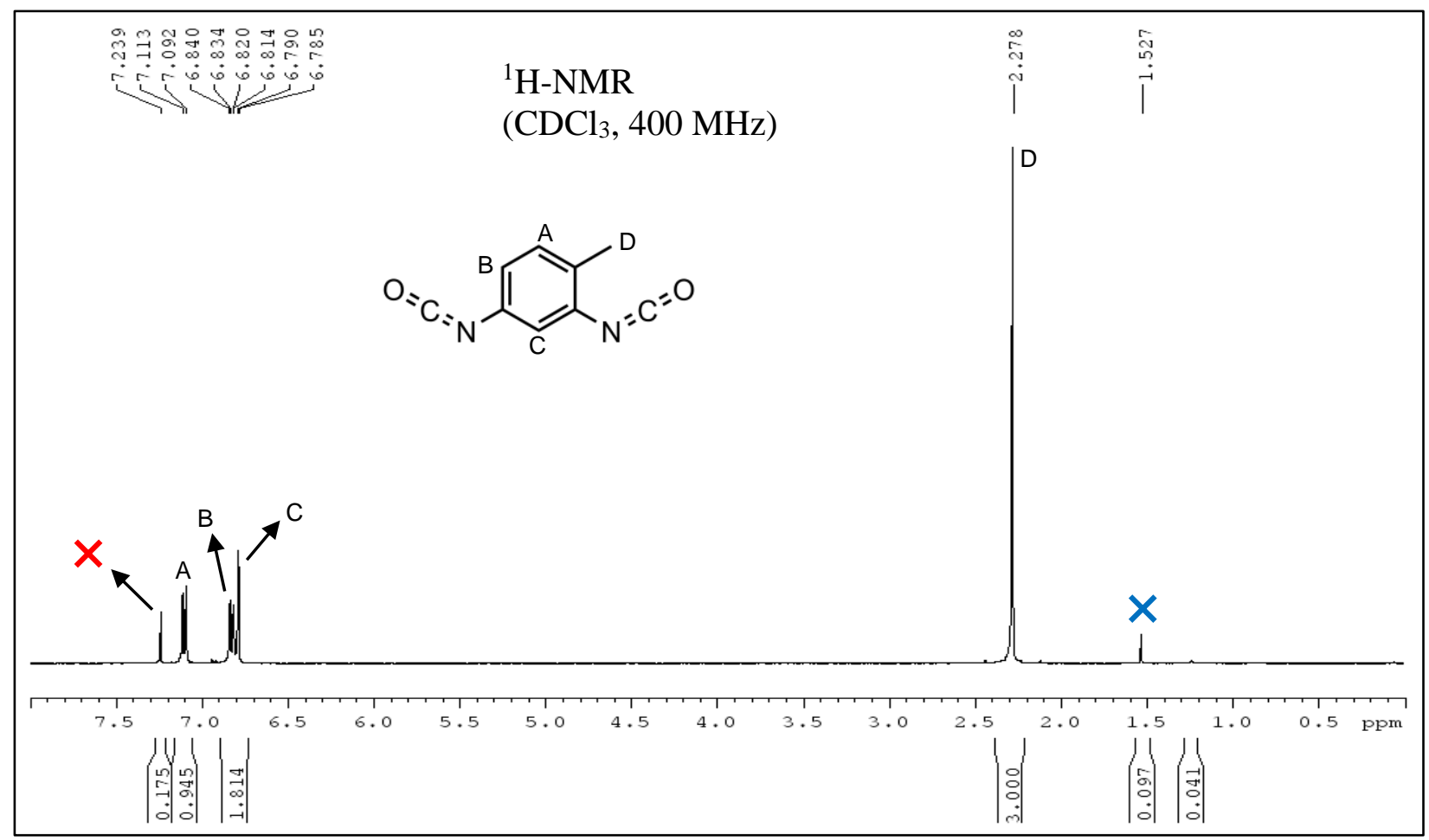

Figure S7. ${ }^{1} \mathrm{H}-\mathrm{NMR}$ spectrum of TDI used for chain extension.

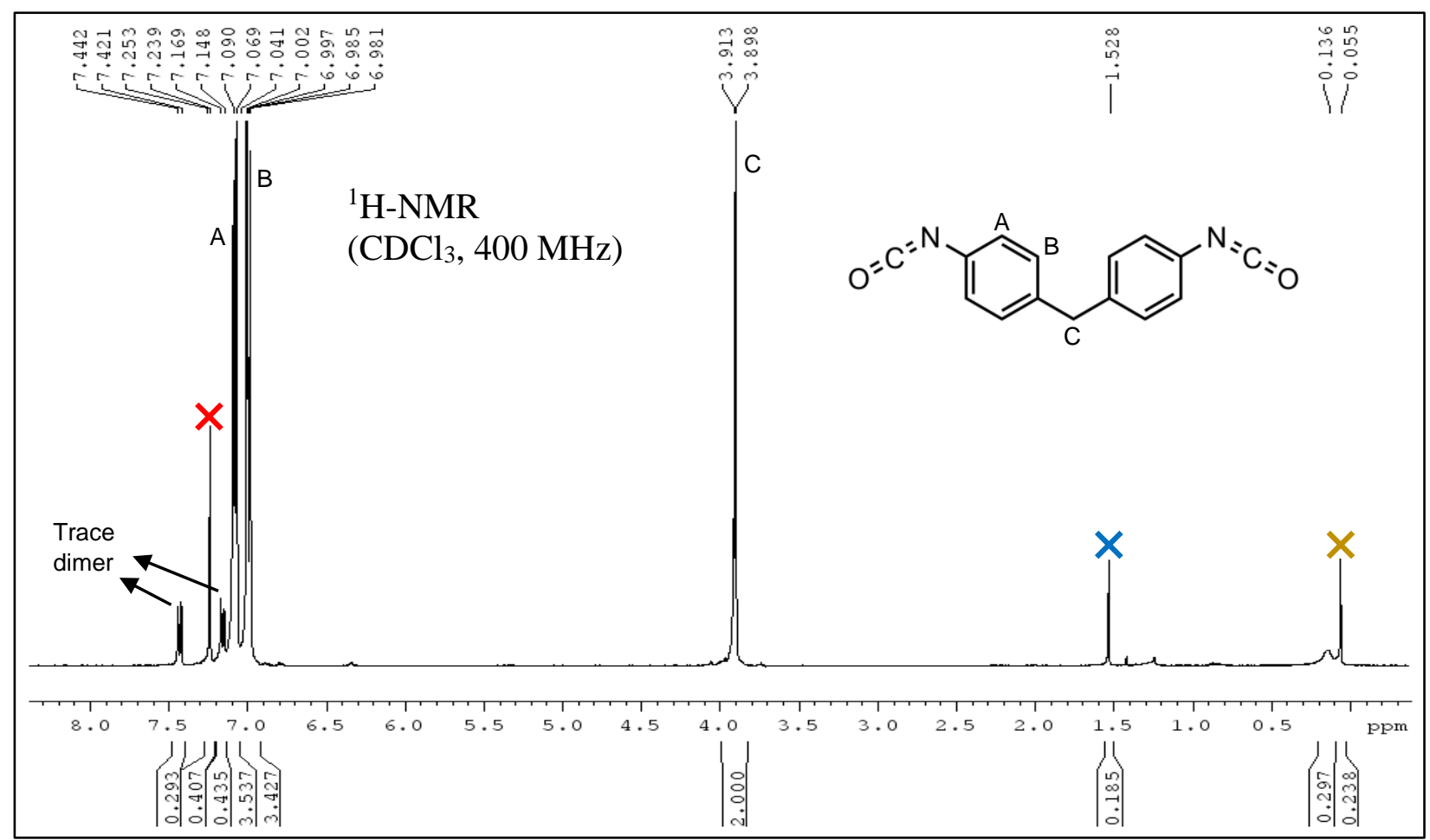

Figure S8. ${ }^{1} \mathrm{H}-\mathrm{NMR}$ spectrum of MDI used for chain extension. 


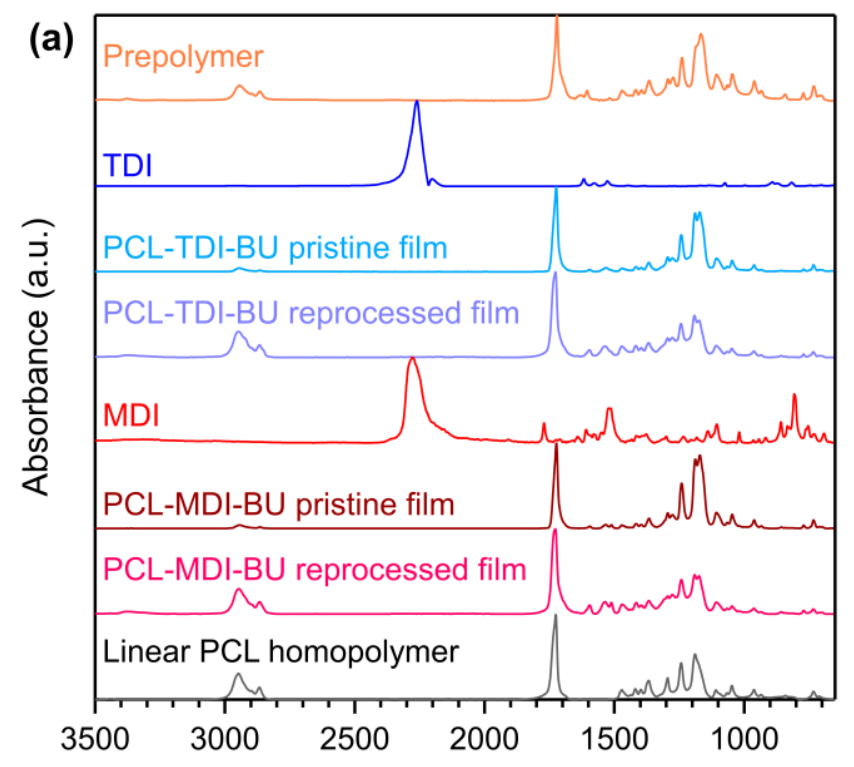

(b)

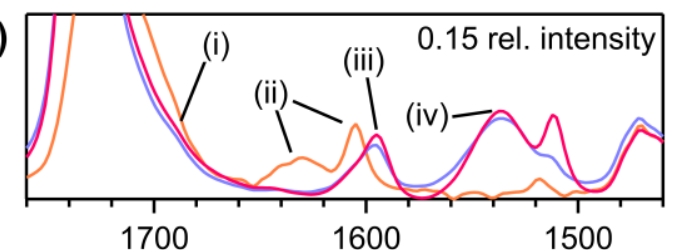

(c)

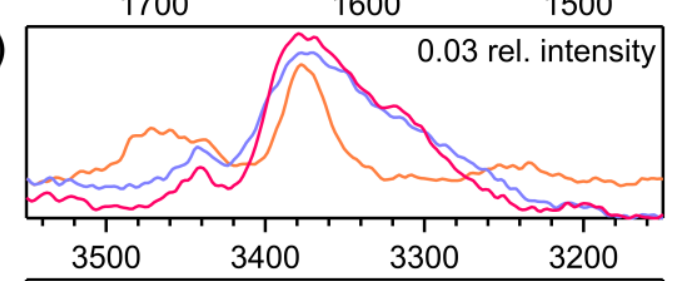

(d)

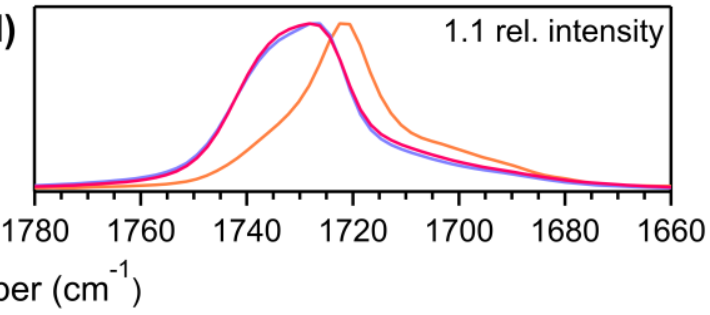

Figure S9. ATR-FTIR spectra of reagents and products: (a) Full spectra, arbitrary normalized intensities; spectra for prepolymer and reprocessed poly(bisurea) films magnified in the (b) $\mathrm{N}-\mathrm{H}$ bending, (c) $\mathrm{N}-\mathrm{H}$ stretching, and (d) $\mathrm{C}=\mathrm{O}$ stretching regions.

In Figure S9a, it can be seen that the diisocyanate $\mathrm{N}=\mathrm{C}=\mathrm{O}$ stretch at $2270-2260 \mathrm{~cm}^{-1}$ is completely absent from the poly(bisurea) films' spectra, indicating completion of the chain extension. Reprocessing slightly reduced film thickness, improving IR signal resolution, and so the reprocessed films' spectra are plotted in Figures S9b-d. The prepolymer's primary amine functionality is evidenced by two peaks, respectively, for asymmetric and symmetric $\mathrm{N}-\mathrm{H}$ bending, 1630 and $1605 \mathrm{~cm}^{-1}$ (b, ii), and two peaks, respectively, for asymmetric and symmetric $\mathrm{N}-\mathrm{H}$ stretching, 3460 and $3374 \mathrm{~cm}^{-1}$ (c). These peaks are blue-shifted by $\sim 10 \mathrm{~cm}^{-1}$ but are consistent in spacing with respect to the corresponding wavenumbers reported for aniline as a model compound for the terminal phenylamine groups. ${ }^{1}$ The PCL C $=\mathrm{O}$ stretch peak (d) is centered at $1724 \mathrm{~cm}^{-1}$ with a wide, low-wavenumber shoulder, which is consistent with literature data. ${ }^{2}$

In contrast, the poly(bisurea) films show peaks at 1595 (b, iii) and $1538 \mathrm{~cm}^{-1}$ (b, iv), which are respectively attributed to the $\mathrm{H}$-bonded urea $\mathrm{C}=\mathrm{O}$ stretch and the urea $(\mathrm{O}) \mathrm{C}-\mathrm{N}-\mathrm{H}$ bend. ${ }^{3,4}$ The peaks are centered at wavenumbers intermediate between those reported for polyurea and 1,3diphenylurea, though an exact correspondence is not expected due to coupling ${ }^{5}$ and a difference in chemical environment. ${ }^{3,6}$ The free urea $\mathrm{C}=\mathrm{O}$ stretch, expected at $\sim 1690 \mathrm{~cm}^{-1}$, appears as a shoulder on the low-wavenumber side $(b, i)$ of the dominant PCL ester $\mathrm{C}=\mathrm{O}$ stretch peak, ${ }^{3}$ though it is difficult to resolve due to the latter's broadness resulting from soft segment H-bonding (d). Free and $\mathrm{H}$-bonded urea $\mathrm{N}-\mathrm{H}$ stretching peaks are respectively expected at $\sim 3450$ and $\sim 3320 \mathrm{~cm}^{-1}$, and the broad convolved peaks extending over this range shown in (c) suggest the coexistence of both IR modes. ${ }^{3,4,7}$ 


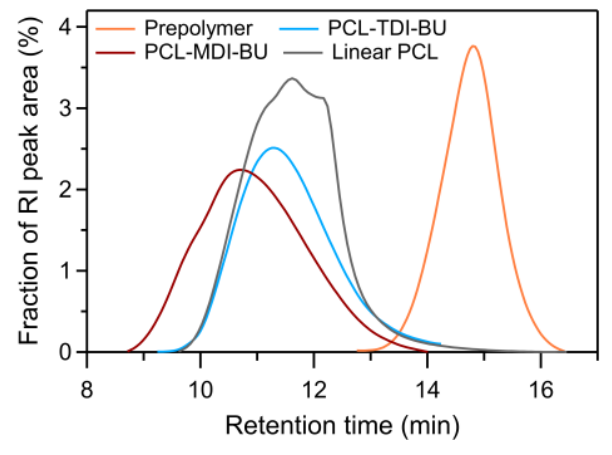

Figure S10. Normalized refractive index curves obtained from GPC for prepolymer, pristine poly(bisurea) films, and linear PCL.

\begin{tabular}{|c|c|c|c|}
\hline Specimen & $\begin{array}{c}M_{\mathrm{n}} \\
\left(\mathrm{kg} \mathrm{mol}^{-1}\right)\end{array}$ & $\begin{array}{c}M_{\mathrm{w}} \\
\left(\mathrm{kg} \mathrm{mol}^{-1}\right)\end{array}$ & PDI \\
\hline Prepolymer & 3.0 & 3.7 & 1.2 \\
\hline PCL-TDI-BU & 44 & 100 & 2.3 \\
\hline PCL-MDI-BU & 76 & 290 & 3.8 \\
\hline Linear PCL & 40 & 90 & 2.2 \\
\hline
\end{tabular}

Table S1. Results of molecular weight analysis obtained using PCL standard calibration curve.

PCL standards for GPC were purchased from Scientific Polymer Products: $M_{\mathrm{n}}=2,270 \mathrm{~g} \mathrm{~mol}^{-1}, M_{\mathrm{w}}$ $=2,380 \mathrm{~g} \mathrm{~mol}^{-1} ; M_{\mathrm{n}}=12,600 \mathrm{~g} \mathrm{~mol}^{-1}, M_{\mathrm{w}}=12,900 \mathrm{~g} \mathrm{~mol}^{-1} ; M_{\mathrm{n}}=39,400 \mathrm{~g} \mathrm{~mol}^{-1}, M_{\mathrm{w}}=41,000 \mathrm{~g}$ $\mathrm{mol}^{-1} ; M_{\mathrm{n}}=87,200 \mathrm{~g} \mathrm{~mol}^{-1}, M_{\mathrm{w}}=96,500 \mathrm{~g} \mathrm{~mol}^{-1} ; M_{\mathrm{n}}=188,100 \mathrm{~g} \mathrm{~mol}^{-1}, M_{\mathrm{w}}=200,700 \mathrm{~g} \mathrm{~mol}^{-1}$.
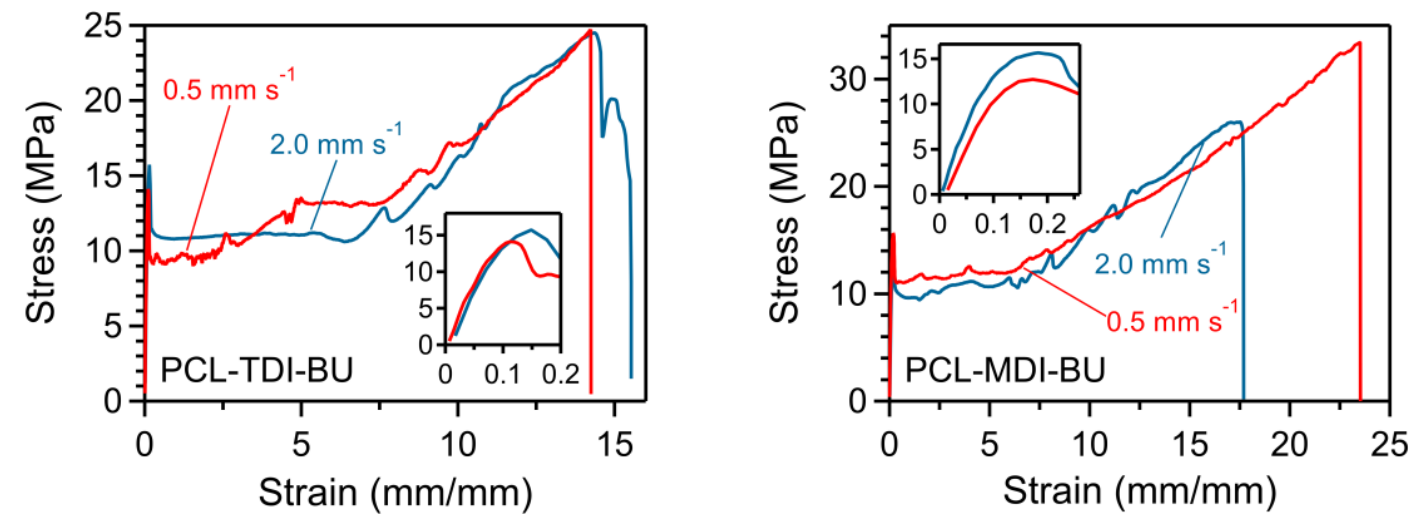

Figure S11. Stress-strain curves for pristine PCL-TDI-BU and PCL-MDI-BU films in strain-tobreak experiments. Insets show magnified neo-Hookean regimes. PCL-TDI-BU's apparent stress peak at $1500 \%$ strain is a slippage artifact. 

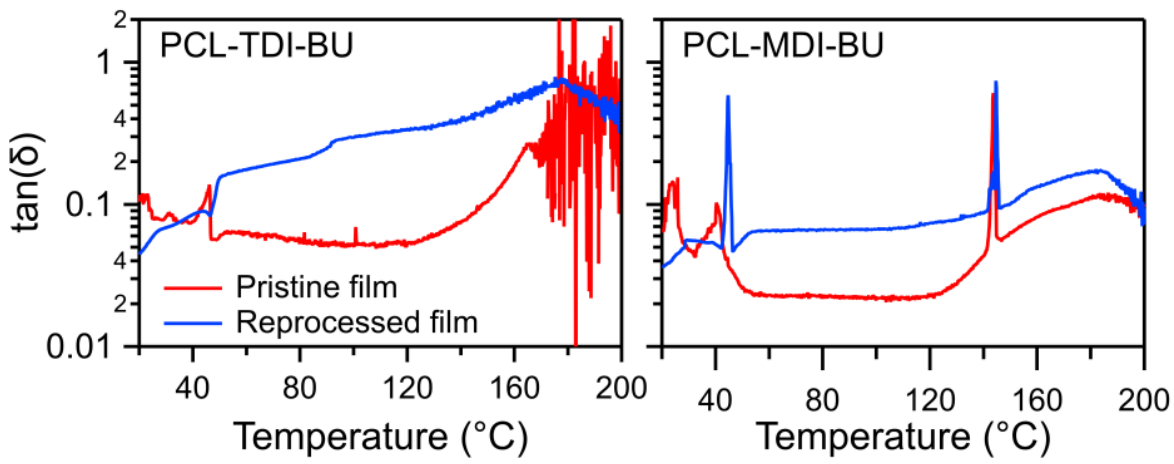

Figure S12. DMA loss tangents for poly(bisurea) thermoplastic elastomers.

Peaks are observed at $T_{\mathrm{m}}$ as expected, and at higher temperatures, the loss tangent plateaus in both pristine and reprocessed samples, enabling elasticity. The reprocessed films were incompletely healed along fragmentation boundaries, causing their loss tangents to plateau at higher values. Between $120^{\circ} \mathrm{C}$ and $140^{\circ} \mathrm{C}$, the loss tangents begin to increase, signaling an increased rate of chain reptation, though the continuity of the stiffness curves is unaffected (main text, Fig. 2). This transition manifests in the abrupt increase in the rate of stress decay (main text, Fig. 3) and permanent strain in a tensile creep experiment (Fig. S18).

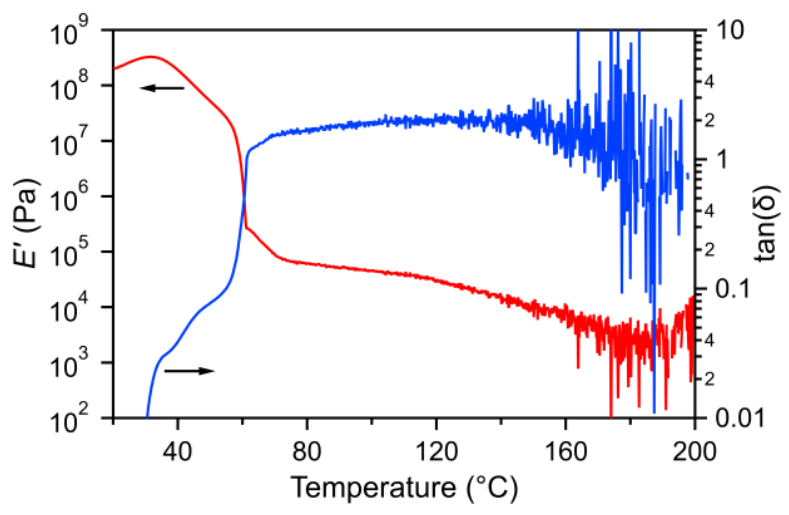

Figure S13. DMA temperature sweep for linear PCL homopolymer. Storage modulus is plotted on the left axis and the loss tangent on the right.

As shown above, the dynamic mechanical behavior of the unfunctionalized PCL homopolymer differs considerably from that of the poly(bisurea)s (main text, Figure 2). The PCL homopolymer lacks a rubbery plateau, melting completely at $60^{\circ} \mathrm{C}$. Though there is an apparent $E^{\prime}$ plateau, the loss tangent is $\sim 2$ for this region, indicating that the crossover point occurs with melting and that the homopolymer behaves as a viscous liquid. In contrast, the poly(bisurea)s exhibited a loss tangent of no greater than $\sim 0.2$ and $E_{\mathrm{r}}$ ' greater than $1 \mathrm{MPa}$ in their rubbery plateau regions, showing thermally stable elastomeric behavior. The increase in noise after the PCL melting temperature is also characteristic of complete melting, as the instrument force transducer's measurement oscillations become large relative to the precision of the force channel signal. 


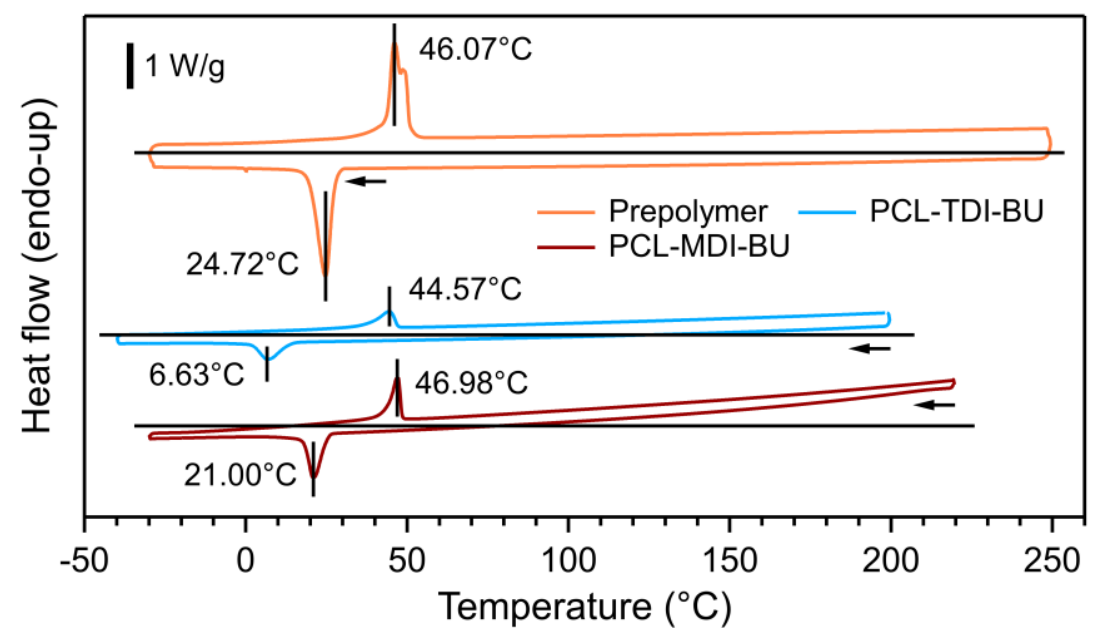

Figure S14. DSC thermal scans of prepolymer and pristine poly(bisurea) films. Each heating scan is the second collected for each sample. Horizontal black lines indicate the axis of zero heat flow for each respective scan. Vertical black lines mark peak maxima and are labeled with the corresponding temperatures. The initial temperature and scanning direction of each trace is denoted by its arrow.

The poly(bisurea)s' melting endotherm occurs at the same temperature as the prepolymer. On cooling, a large thermal hysteresis is observed. For the poly(bisurea)s, the degree of undercooling required for recrystallization of the PCL soft segments increases, which is consistent with hardsoft segmental phase mixing resulting in the softening of the crystalline state seen in DMA experiments. Additionally, compared to the PCL prepolymer, the poly(bisurea)s display a more pronounced increase in heat capacity during heating, which causes continued endothermic heat flow at high temperatures even during the cooling scan. 

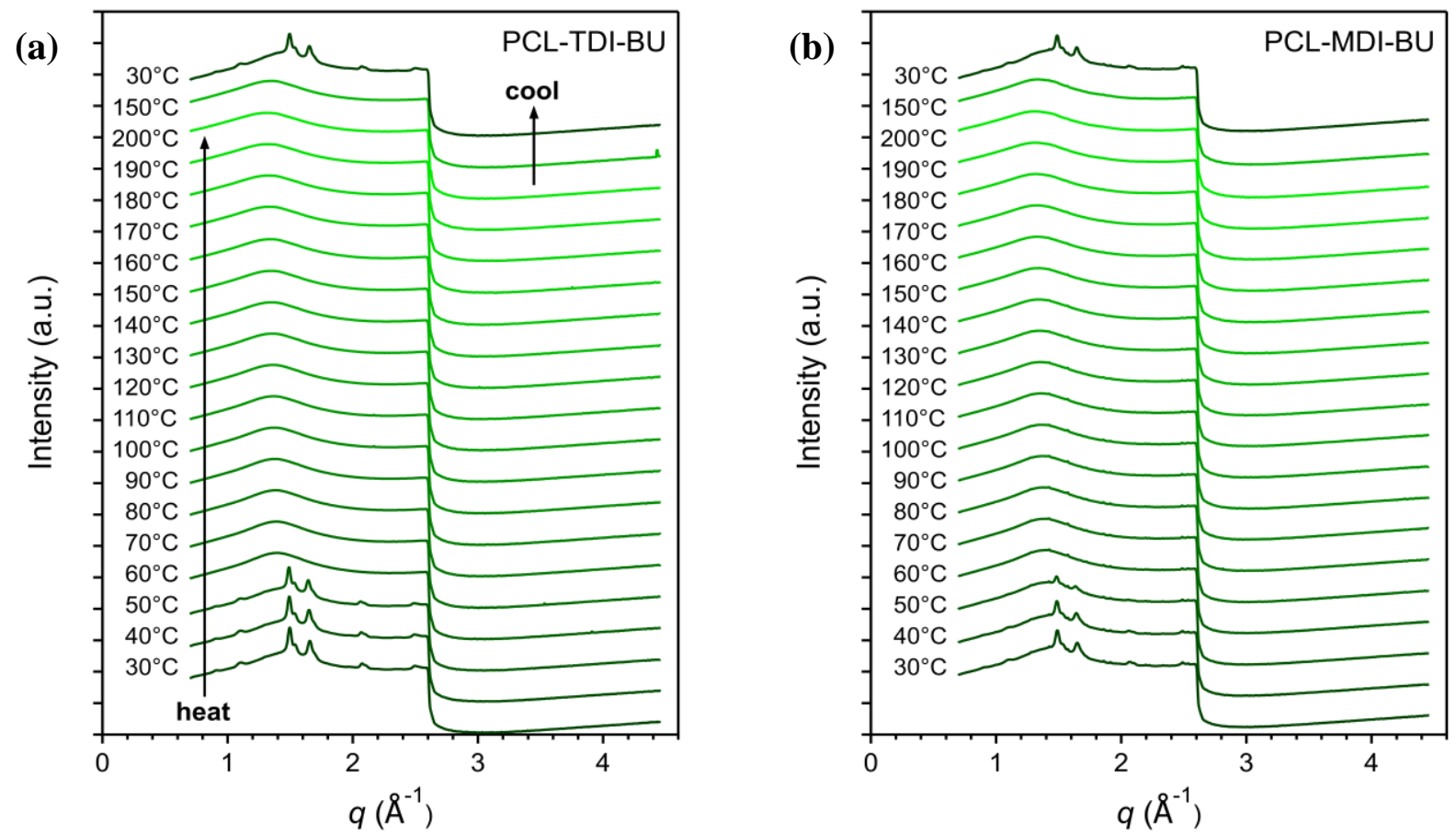

Figure S15. Temperature-resolved 1D wide-angle X-ray scattering pattersn for pristine (a) PCLTDI-BU and (b) PCL-MDI-BU films. Arbitrary intensity is plotted on a logarithmic scale.
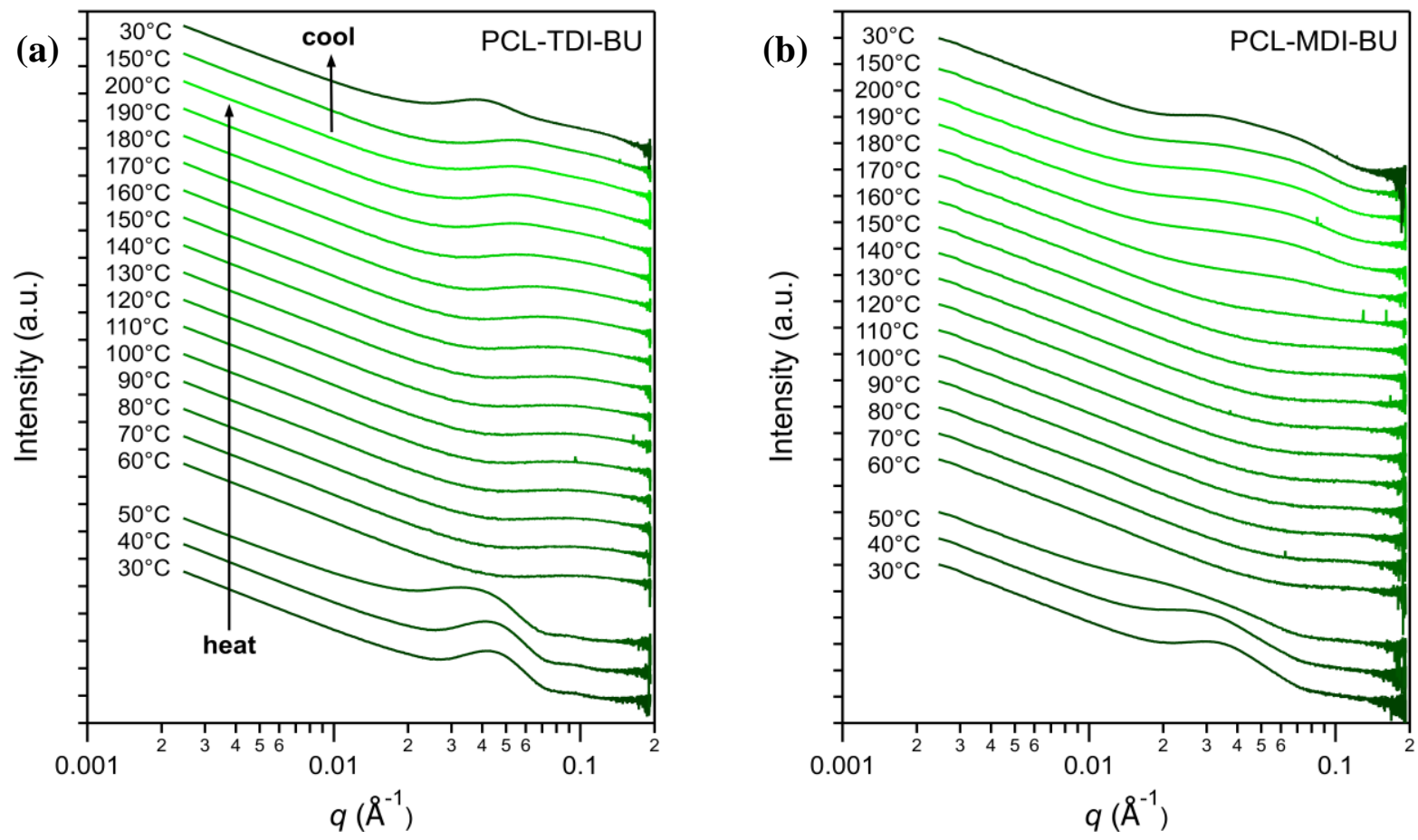

Figure S16. Temperature-resolved 1D small-angle X-ray scattering patterns for pristine (a) PCLTDI-BU and (b) PCL-MDI-BU films. Arbitrary intensity is plotted on a logarithmic scale. 

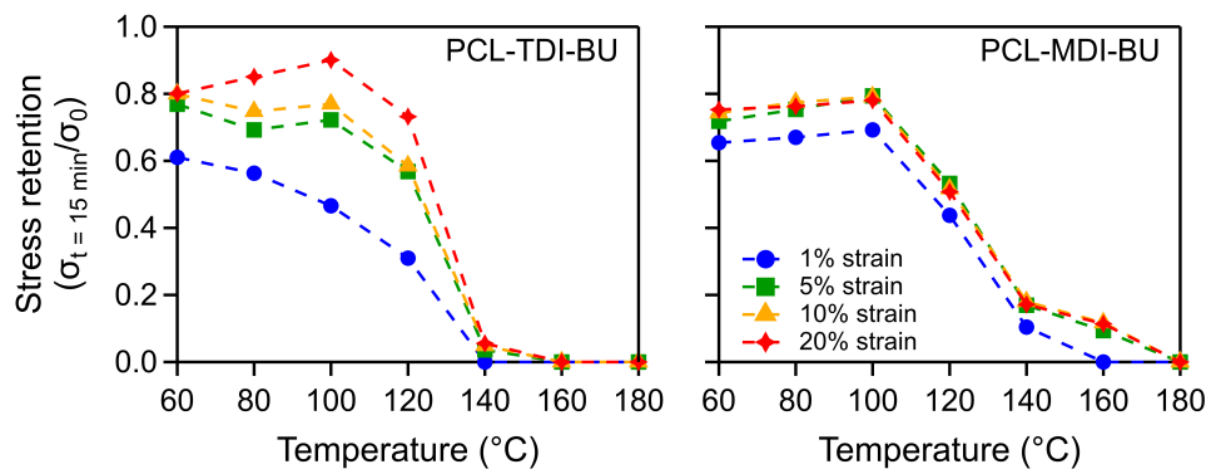

Figure S17. Normalized stress retention in 15-min stress relaxation experiments conducted at various temperatures and strains for pristine poly(bisurea) films.
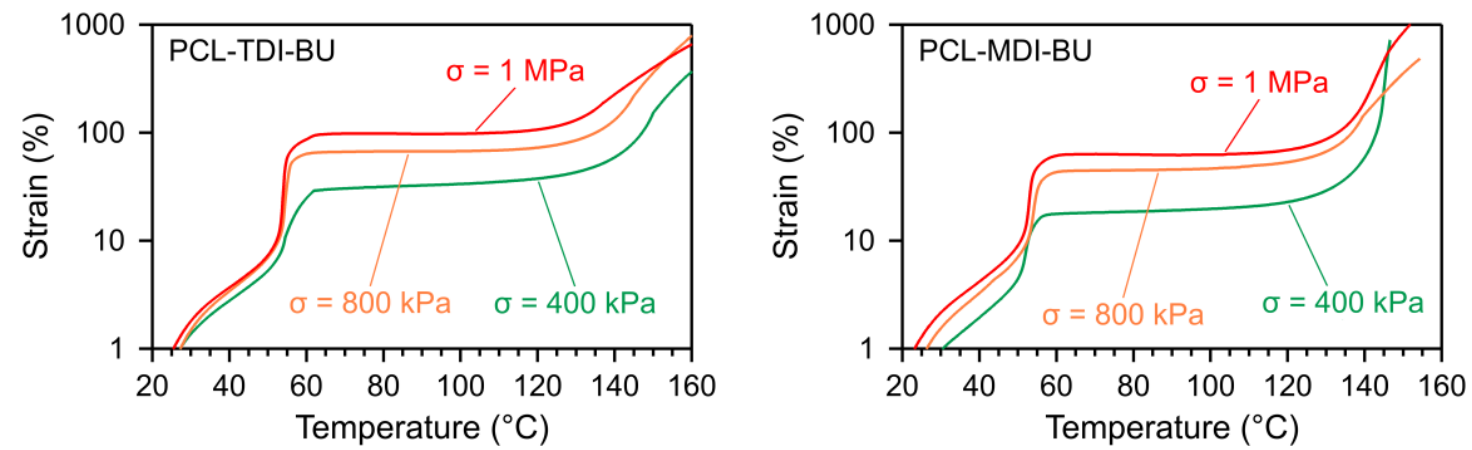

Figure S18. Tensile creep experiments for poly(bisurea) films. Temperature was ramped linearly at $1^{\circ} \mathrm{C} \mathrm{min}^{-1}$. Nominal stress loadings are indicated in the plots.

In the creep traces above, the melting of the PCL soft segments at $T_{\mathrm{m}}$ is evident from the rapid increase in sample strain by approximately one order of magnitude. In parallel with the $E_{\mathrm{r}}$ ' plateaus for temperatures above $T_{\mathrm{m}}$, here, the constant-stress strain exhibits a plateau. Strain then begins increasing rapidly between $120^{\circ} \mathrm{C}$ and $140^{\circ} \mathrm{C}$, consistent with the increase in the loss tangent signaling an increased rate of chain reptation.

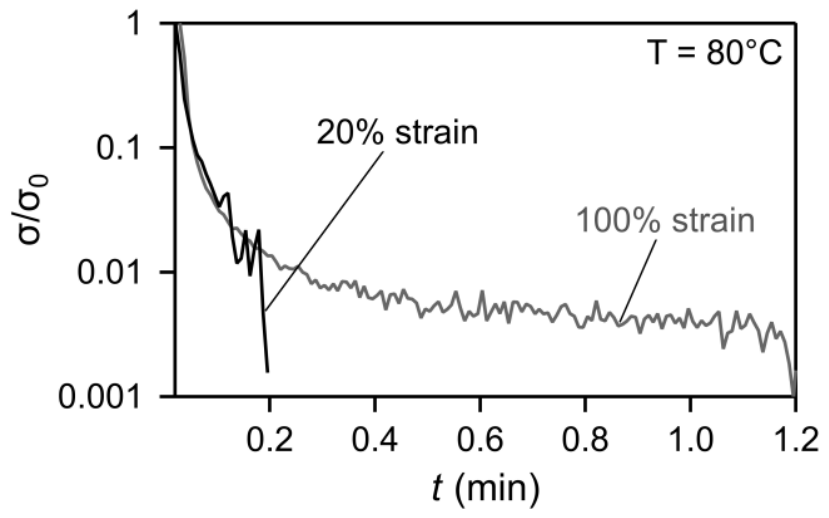

Figure S19. Normalized stress relaxation curve for linear PCL at $80^{\circ} \mathrm{C}$ and various strains. 

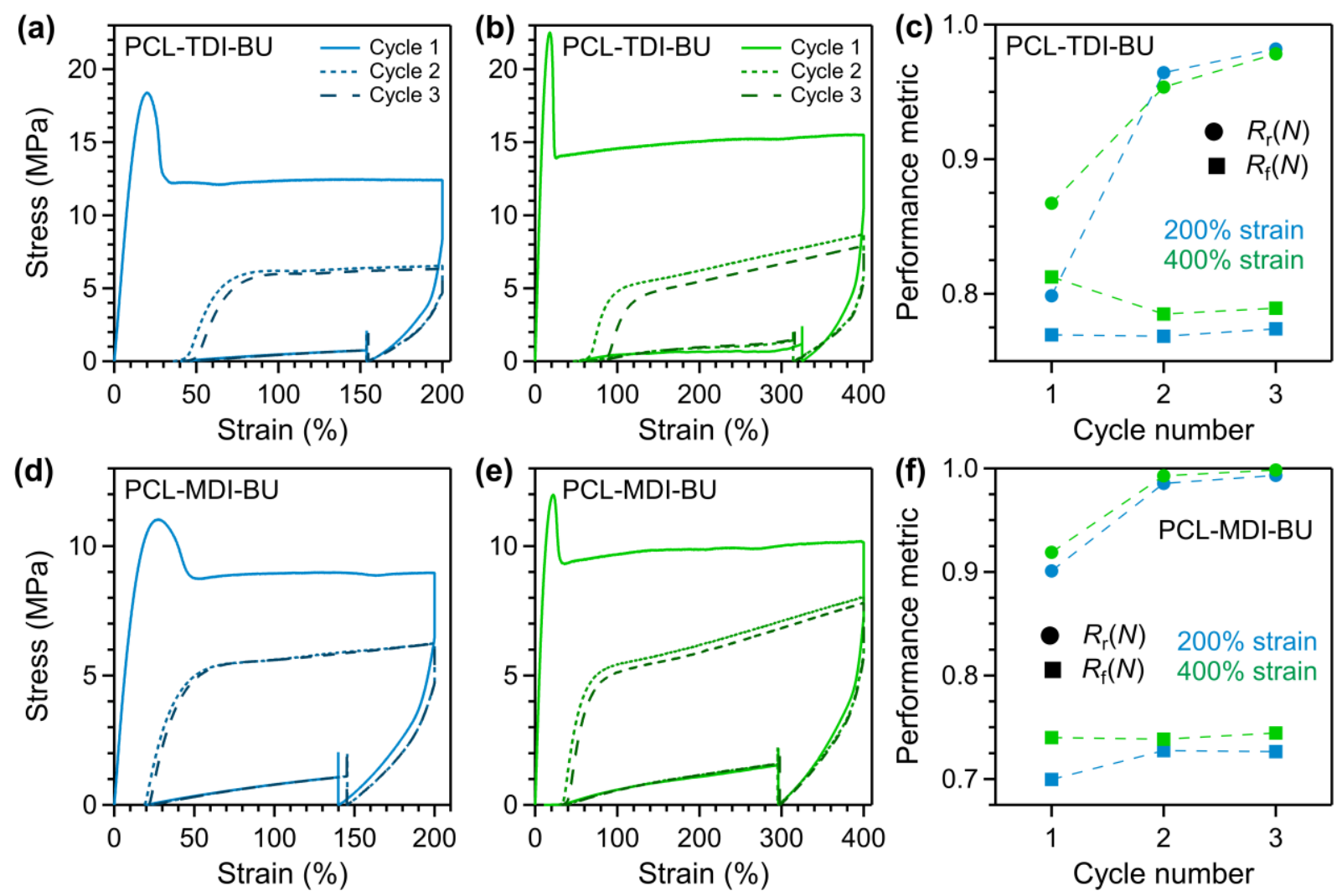

Figure S20. Cold-draw shape-memory of pristine poly(bisurea) films: shape-memory cycles of (a) PCL-TDI-BU with $200 \%$ and (b) PCL-TDI-BU with $400 \%$ peak strain, (c) PCL-TDI-BU SM performance metrics; (d) SM curves, PCL-MDI-BU with $200 \%$ and (e) $400 \%$ peak strain, (f) PCLMDI-BU SM performance metrics.

Cold-draw stress-strain curves are shown in Figures S20a, S20b, S20d, and S20e. After training in the first cycle, the sharp stress peak associated with the yielding of the untrained semicrystalline segments disappears, and the stress initially imparted at peak strain decreased by up to a factor of $2 / 3$. Thereafter, stress relaxation before unloading is smaller than that observed in the first cycle, and the unloading and recovery curves trace each other closely, further indicating that weak, elastically non-ideal network crosslinks are destroyed in the first cycle. Strain fixity ratios and shape recovery ratios are displayed in Figures S20c and S20f. Stress decay in the deformed state resulting from reconfiguration of semicrystalline PCL domains below $T_{\mathrm{m}}$ is much slower than that from recrystallization from a melted state, and so cold-draw fixities were appreciably lower than those obtained from hot-draw experiments. Strain recovery ratios were again excellent, rising to $\geq 95 \%$ after training and increasing again in the third cycle. 
Table S2. Shape fixity ratios, $R_{\mathrm{f}}(N)$, for hot-drawn PCL-TDI-BU poly(bisurea) films. The corresponding cycles are shown in main-text Figure 4 and Figure S21 below.

\begin{tabular}{|c|c|c|c|c|c|c|}
\hline & \multicolumn{3}{|c|}{ Pristine films } & \multicolumn{3}{c|}{ Reprocessed films } \\
\hline Peak strain & Cycle 1 & Cycle 2 & Cycle 3 & Cycle 1 & Cycle 2 & Cycle 3 \\
\hline $25 \%$ & 0.957 & 0.932 & - & 0.982 & 0.982 & - \\
\hline $50 \%$ & 1.00 & 1.00 & - & 1.00 & 1.00 & 0.998 \\
\hline $100 \%$ & 1.00 & 0.991 & - & - & - & - \\
\hline $200 \%$ & 1.00 & 1.00 & 1.00 & - & - & - \\
\hline
\end{tabular}

Table S3. Shape fixity ratios, $R_{\mathrm{f}}(N)$, for hot-drawn PCL-MDI-BU poly(bisurea) films. The corresponding cycles are shown in main-text Figure 4 and Figure S21 blow.

\begin{tabular}{|c|c|c|c|c|c|c|}
\hline & \multicolumn{3}{|c|}{ Pristine films } & \multicolumn{3}{c|}{ Reprocessed films } \\
\hline Peak strain & Cycle 1 & Cycle 2 & Cycle 3 & Cycle 1 & Cycle 2 & Cycle 3 \\
\hline $25 \%$ & 0.982 & 0.943 & - & 0.919 & 0.888 & - \\
\hline $50 \%$ & 1.00 & 1.00 & - & 0.937 & 0.928 & 0.925 \\
\hline $100 \%$ & 1.00 & 1.00 & - & - & - & - \\
\hline $200 \%$ & 1.00 & 1.00 & 1.00 & - & - & - \\
\hline
\end{tabular}
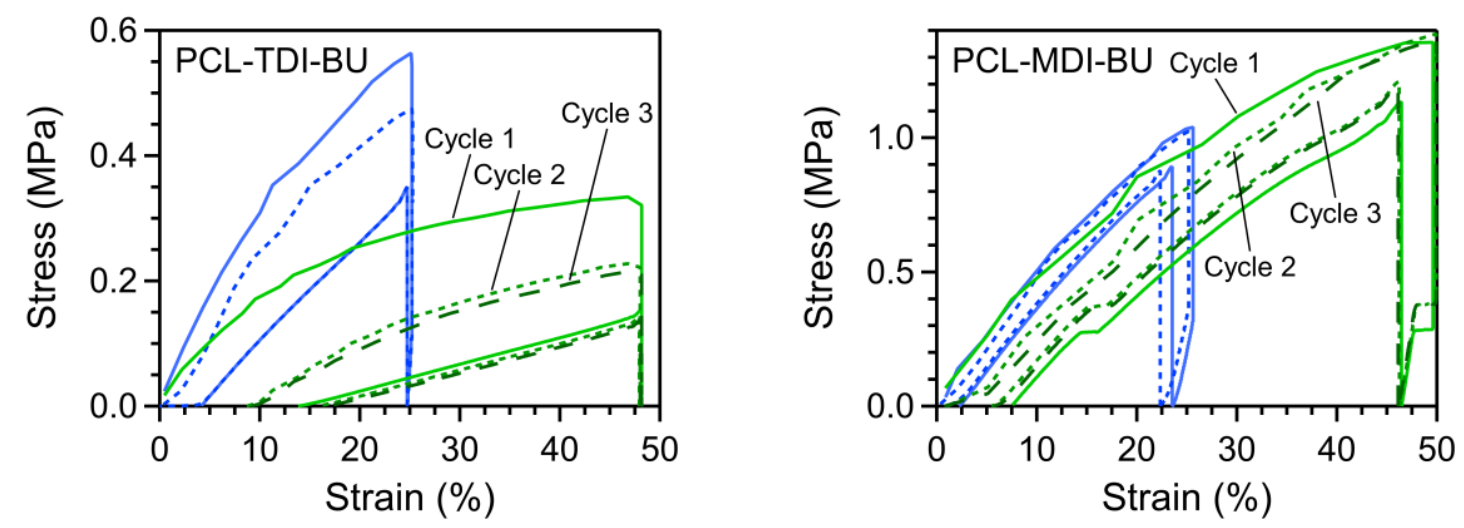

Figure S21. Hot-draw shape-memory cycles for reprocessed poly(bisurea) films. Peak strains could not exceed $50 \%$ due to weakening along fragmentation boundaries in the reprocessed specimens. The weakening was also responsible for the yielding of the PCL-TDI-BU film during initial loading in the 50\%-strain trials, which effectively softened the sample and caused a large deviation from the load curve obtained with $25 \%$ strain. In contrast, the loading curves of the reprocessed PCL-MDI-BU films are consistent as in the pristine film cycles due to more complete fragment annealing during the reprocessing procedure. 

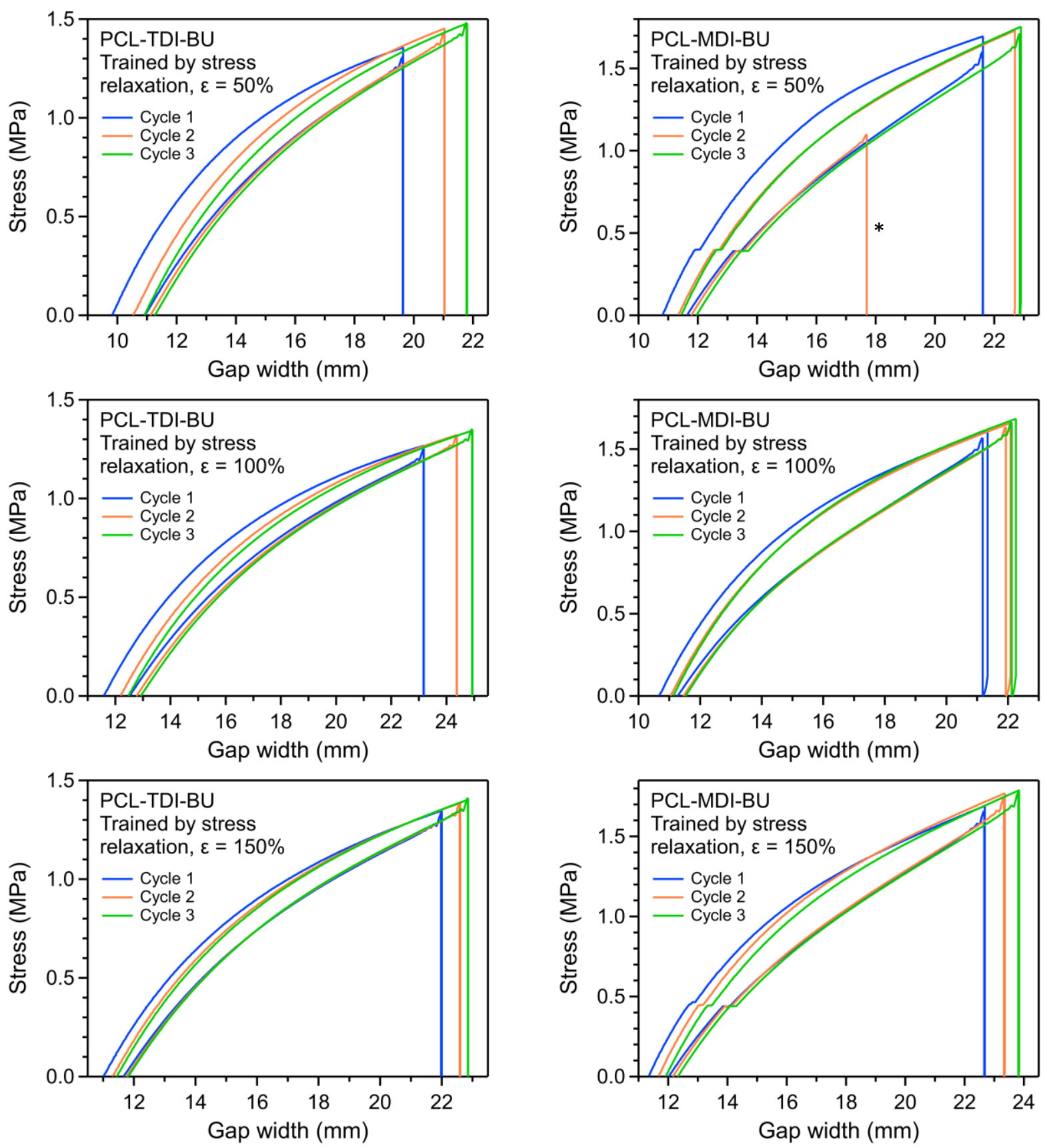

Figure S22. Shape-memory cycling data for pristine poly(bisurea) films pre-trained through stress relaxation at various strains. The peak strain in all cycles was $100 \%$. To account for the effects of creep training, axial force was zeroed in between each cycle by increasing the initial gap, and so the strain basis is different for each cycle; hence, elongation is reported in terms of absolute gap width. In the top-right plot, an asterisk (*) denotes where the RSA-G2 force channel failed to respond to zero load and halt post-recrystallization unloading. This resulted in a smaller gap width measured upon re-heating and the reemergence of stress, but because the sample was still under tension after re-heating, the slow final unload still gave an accurate shape recovery ratio. 

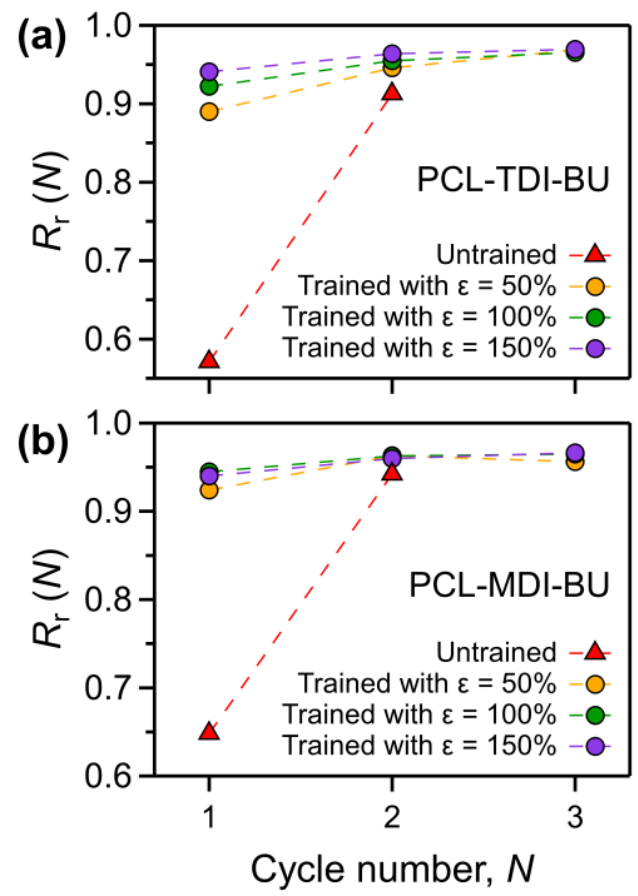

Figure S23. Shape recovery ratios achieved in hot-draw thermomechanical cycling by pristine (a) PCL-TDI-BU and (b) PCL-MDI-BU films pre-trained through stress relaxation at various strains. All shape-memory cycles were conducted at $100 \%$ strain. The untrained pristine film recoveries for $100 \%$ strain are reproduced from main-text Figures $4 \mathrm{c}$ and $4 \mathrm{~d}$ for comparison.

Table S4. Shape-memory performance metrics for pristine, pre-trained PCL-TDI-BU films. The corresponding cycles are shown above in Figure S23a.

\begin{tabular}{|c|c|c|c|c|c|c|}
\hline $\begin{array}{c}\text { Stress relaxation } \\
\text { training condition }\end{array}$ & \multicolumn{2}{|c|}{$\varepsilon=50 \%$} & \multicolumn{2}{c|}{$\varepsilon=100 \%$} & \multicolumn{2}{c|}{$\varepsilon=150 \%$} \\
\hline Cycle number, $N$ & $R_{\mathrm{f}}(N)$ & $R_{\mathrm{r}}(N)$ & $R_{\mathrm{f}}(N)$ & $R_{\mathrm{r}}(N)$ & $R_{\mathrm{f}}(N)$ & $R_{\mathrm{r}}(N)$ \\
\hline 1 & 1.00 & 0.890 & 1.00 & 0.922 & 1.00 & 0.941 \\
\hline 2 & 1.00 & 0.946 & 1.00 & 0.955 & 0.998 & 0.964 \\
\hline 3 & 1.00 & 0.969 & 1.00 & 0.966 & 0.999 & 0.969 \\
\hline
\end{tabular}

Table S5. Shape-memory performance metrics for pristine, pre-trained PCL-MDI-BU films. The corresponding cycles are shown above in Figure S23b.

\begin{tabular}{|c|c|c|c|c|c|c|}
\hline $\begin{array}{c}\text { Stress relaxation } \\
\text { training condition }\end{array}$ & \multicolumn{2}{|c|}{$\varepsilon=50 \%$} & \multicolumn{2}{c|}{$\varepsilon=100 \%$} & \multicolumn{2}{c|}{$\varepsilon=150 \%$} \\
\hline Cycle number, $N$ & $R_{\mathrm{f}}(N)$ & $R_{\mathrm{r}}(N)$ & $R_{\mathrm{f}}(N)$ & $R_{\mathrm{r}}(N)$ & $R_{\mathrm{f}}(N)$ & $R_{\mathrm{r}}(N)$ \\
\hline 1 & 1.00 & 0.924 & 0.986 & 0.945 & 1.00 & 0.940 \\
\hline 2 & 0.999 & 0.963 & 0.987 & 0.963 & 0.999 & 0.960 \\
\hline 3 & 0.997 & 0.956 & 0.988 & 0.965 & 0.998 & 0.966 \\
\hline
\end{tabular}




\section{Experimental Methods}

Materials and synthetic protocol. Shape-memory thermoplastics were synthesized via stoichiometric reaction of a custom diphenylamine-terminated poly( $\varepsilon$-caprolactone) (PCL) prepolymer (Scientific Polymer, 3,180 g mol-1) and diisocyanates MDI (4,4'-methylene diphenyl diisocyanate, Sigma-Aldrich, 98\%) or TDI (toluene-2,4-diisocyanate, TCI Chemicals, >98\%). Linear PCL ( $\mathrm{Mn}=75,475 \mathrm{~g}$ mol-1 nominal, acid endcap) was purchased from PolySciTech. The prepolymer was melted at $65{ }^{\circ} \mathrm{C}$ on a glass plate covered with a PTFE-coated aluminum adhesive layer and degassed in a vacuum oven $\left(\mathrm{T}=65^{\circ} \mathrm{C}\right)$ for 10 minutes. A stoichiometric amount of diisocyanate was melted and hand-mixed with the prepolymer for 30 seconds. The reaction mixture was then de-gassed for 10 minutes under vacuum. A PTFE spacer was placed around the reaction mixture, and a second, preheated PTFE-coated glass plate was placed on top of the mixture. The film and assembly were cured at $65^{\circ} \mathrm{C}$ for three days.

Structural and Thermal Characterization. 1H-NMR spectra were collected using a Brüker 400MHz spectrometer. ATR-IR spectra were collected using a Bruker Tensor 27 spectrometer. X-ray data were taken on a diffractometer (Rigaku Synergy-S) using Mo K $\alpha$ radiation $(50 \mathrm{kV}, 1 \mathrm{~mA})$. Polymer molecular weights were deter-mined using a Dionex system (Ultimate 3000) configured for size exclusion chromatography (SEC) - equipped with an isocratic pump, autosampler, two gel columns (Shodex KF-402.5HQ and KF-404HQ), and refractive index detector (ERC, Model RefractoMax520). Polymer films were dissolved into tetrahydrofuran (THF) and filtered through a $0.45 \mathrm{~mm}$ membrane. Molecular weight and dispersity were calculated by comparison to a calibration curve generated using the PCL standards. Differential scanning calorimetry was conducted using a commercial calorimeter (Q2000, TA Instruments).

$X$-ray scattering. X-ray scattering experiments were performed at Argonne National Lab using Sector 5 of the Advanced Photon Source (APS). Square fragments were cut from pristine films and stacked in aluminum DSC pans. The pans were then sealed under inert atmosphere in a glovebox. In each scattering experiment, patterns were recorded at $10-^{\circ} \mathrm{C}$ intervals as the sample was heated from $30^{\circ} \mathrm{C}$ to $200^{\circ} \mathrm{C}$, and then at $150^{\circ} \mathrm{C}$ and $30^{\circ} \mathrm{C}$ as the sample was cooled. Samples were annealed prior to beam exposure for the following time intervals: 5 minutes for $30^{\circ} \mathrm{C} \leq T \leq$ $90^{\circ} \mathrm{C}, 30$ minutes for $100^{\circ} \mathrm{C} \leq T \leq 180^{\circ} \mathrm{C}$, and 5 minutes for $T=190,200,150,30^{\circ} \mathrm{C}$. Patterns were acquired with $\lambda=0.7293 \AA$ and an exposure time of $1.0 \mathrm{~s}$.

Film Reprocessing. Pristine films were cut into mm-sized fragments using a razor blade. Fragments were sandwiched between two PTFE spacers and a mold frame with a 2- x 5-cm void. The assembly was sandwiched be-tween two $6 \times 6$ " stainless steel plates, which were placed in a Melt Press (Carver, Model 5420) set to $180^{\circ} \mathrm{C}$. To maintain thermal contact, the plate gap was gradually reduced as fragments melted, and once the material was flattened, the plates were compressed with minimal measured pressure for five minutes. The temperature set-point was then reduced to room temperature, and the reprocessed film was allowed to cool before extraction. 
Thermomechanical Characterization. Stress relaxation, DMA, and hot-draw shape-memory experiments were conducted on rectangular film samples with an approximate size of $3 \mathrm{~mm} \times 25$ mm using a TA Instruments RSA-G2 Solids Analyzer. Cold-draw shape-memory and strain-tobreak experiments were conducted on dog-bone film samples (3.380 mm width, $7.620 \mathrm{~mm}$ gage length) using a QT/5 MTS tensile testing frame. Step-by-step stress relaxation, shape-memory and strain-to-break procedures are detailed below.

Stress Relaxation. Samples were heated to the experimental temperature and drawn to the desired peak strain at an extension rate ranging from 0.5-5.0 $\mathrm{mm} \mathrm{s}^{-1}$, depending on the absolute extension required to achieve the target strain. This precaution was taken to minimize overshoot, which was unavoidable even after PID-tuning the RSA-G2 device in cases where the target strain could be achieved in less than $100 \mathrm{~ms}$ with the nominal extension rate.

Dynamic Mechanical Analysis. All DMA experiments were conducted using an oscillatory frequency of $6.28 \mathrm{rad} \mathrm{s}^{-1}$, an oscillation strain of $0.6 \%$, and a linear temperature ramp rate of $1{ }^{\circ} \mathrm{C}$ $\min ^{-1}$. For pristine, un-trained poly(bisurea) films, significant creep was observed at the shapememory triggering temperature. This resulted in the films being under compression the remainder of the experiments and worsened the feature-to-noise ratio near the flow temperature. To remedy this, the films were conditioned through the following procedure: (1) ramp from $20^{\circ} \mathrm{C}$ to $60^{\circ} \mathrm{C}$ with oscillation; (2) isothermally extend to a peak load of $1 \mathrm{MPa}$ at $60^{\circ} \mathrm{C}$; (3) isothermal 20-min stress relaxation at $60^{\circ} \mathrm{C}$; (4) cool from $60^{\circ} \mathrm{C}$ to $20^{\circ} \mathrm{C}$ at $60{ }^{\circ} \mathrm{C} \mathrm{min}$ min $^{-1}$ at constant strain; (5) hold isothermally at $20^{\circ} \mathrm{C}$ for 30 minutes to allow for recrystallization; and (6) ramp from $20^{\circ} \mathrm{C}$ to $200^{\circ} \mathrm{C}$ with oscillation (experimental trace). Steps 2-4 fixed the sample in a deformed state, causing a shape-memory recovery at the triggering temperature during the experimental trace, manifesting in a sharp stiffening peak in storage modulus occurring after the initial drop. The modulus then settled to the tensed plateau value as desired.

Hot-Draw Shape-Memory Cycling. The poly(bisurea) films were heated to $60^{\circ} \mathrm{C}$ and analyzed according to the following protocol: (1) isothermal drawing to the targeted peak strain at an extension rate ranging from $0.5-2.0 \mathrm{~mm} \mathrm{~s}^{-1}$ to minimize overshoot as described previously; (2) isothermal 20-min stress relaxation to allow for training and minimize active relaxation in subsequent steps; (3) cooling from $60^{\circ} \mathrm{C}$ to $25^{\circ} \mathrm{C}$ at $60{ }^{\circ} \mathrm{C} \mathrm{min}{ }^{-1}$ followed by equilibration to recrystallize, 40 minutes total; (4) isothermal unloading at $0.005 \mathrm{~mm} \mathrm{~s}^{-1}$ only if full stress decay did not occur during Step 3 (if so, proceed immediately to Step 5); (5) temperature ramp from $25^{\circ} \mathrm{C}$ to $60^{\circ} \mathrm{C}$ at $60{ }^{\circ} \mathrm{C} \mathrm{min}{ }^{-1}$ for 35 seconds; and (6) isothermal unloading at $0.01 \mathrm{~mm} \mathrm{~s}^{-1}$ to zero stress.

Cold-Draw Shape-Memory Cycling. Thermal control on the open-air MTS device was enabled by a custom polyimide-insulated box retrofitted to the testing stage and allowing a maximum extension of $120 \mathrm{~mm}$. A heating pad controlled with a PID-tuned Cole-Parmer temperature controller was placed upright around the sample, with a K-type thermocouple inserted through the upper opening of the box and positioned as close to the sample as possible. A 12.0-V computer fan powered by a DC power supply was also placed inside the box to convectively drive airflow 
and speed sample equilibration. The chamber was sealed at all points during experimental trials. Poly(bisurea) films were analyzed using a 50-N load cell according to the following protocol with $0.02 \mathrm{~mm} \mathrm{~s}^{-1}$ used for all extension and unload rates: (1) isothermal drawing to target peak strain at room temperature; (2) isothermal 1-hr stress relaxation at room temperature; (3) isothermal unloading to zero stress at room temperature; (4) temperature ramping from room temperature to $60^{\circ} \mathrm{C}$ and equilibration, 10 minutes total; (5) isothermal unloading to zero stress at $60^{\circ} \mathrm{C}$. In between each shape-memory cycle, the heating pad was deactivated and the box was opened, and the sample was allowed to cool and recrystallize for 90 minutes in open air with the fan still operative.

Strain-to-Break Experiments. The tensile data reported here are representative of the trials with minimal slippage of the samples. This was achieved through the use of thin samples $(75-\mu \mathrm{m}$ thickness for PCL-MDI-BU and 250- $\mu \mathrm{m}$ thickness for PCL-TDI-BU) and standard serrated MTS grips. Films were analyzed using a 1250-N load cell and drawn at room temperature. 


\section{References}

1. Chinnappan, A.; Kim, H., Transition metal based ionic liquid (bulk and nanofiber composites) used as catalyst for reduction of aromatic nitro compounds under mild conditions. $R s c A d v$ 2013, 3 (10), 3399-3406.

2. Lyu, J. S.; Lee, J. S.; Han, J., Development of a biodegradable polycaprolactone film incorporated with an antimicrobial agent via an extrusion process. Sci Rep-Uk 2019, 9.

3. Jiang, S.; Shi, R. H.; Cheng, H. Y.; Zhang, C.; Zhao, F. Y., Synthesis of polyurea from 1,6-hexanediamine with $\mathrm{CO}_{2}$ through a two-step polymerization. Green Energy Environ 2017, 2 (4), 370-376.

4. Yamamoto, T.; Shibayama, M.; Nomura, S., Structure and Properties of Fatigued Segmented Poly(Urethaneurea)S .3. Quantitative-Analyses of Hydrogen-Bond. Polym J 1989, 21 (11), 895-903.

5. Keuleers, A.; Desseyn, H. O.; Rousseau, B.; Van Alsenoy, C., Vibrational analysis of urea. J Phys Chem A 1999, 103 (24), 4621-4630.

6. Inaloo, I. D.; Majnooni, S., $\mathrm{A} \mathrm{Fe}_{3} \mathrm{O}_{4} @ \mathrm{SiO}_{2} / \mathrm{Schiff} \mathrm{Base} / \mathrm{Pd}$ Complex as an Efficient Heterogeneous and Recyclable Nanocatalyst for One-Pot Domino Synthesis of Carbamates and Unsymmetrical Ureas. Eur J Org Chem 2019, 2019 (37), 6359-6368.

7. Teo, L. S.; Chen, C. Y.; Kuo, J. F., Fourier transform infrared spectroscopy study on effects of temperature on hydrogen bonding in amine-containing polyurethanes and poly(urethane-urea)s. Macromolecules 1997, 30 (6), 1793-1799. 